\title{
Optimization of electric heating operation based on day-ahead electricity spot market
}

\author{
Chengyuan $\mathrm{Li}^{1, *}$, Limin Jang ${ }^{2}$, and Yu Qin ${ }^{1}$ \\ ${ }^{1}$ China Electric Power Research Institute, 100192 Beijing, China \\ ${ }^{2}$ China Electric Power Research Institute, 100192 Beijing, China
}

\begin{abstract}
The emergence of Electricity Spot Market makes it possible that distributed electric heating equipment connect with the grid and participate in market competition as well. However, due to the functional limits, it is impossible for the power supply side to have a thorough understanding of the specific conditions of the load characteristics of the power user, and the user side will not take a global consideration of the real-time security and stability of the power grid. This paper focuses on the research about electricity consumption strategy of thermoelectric coupled load with the background of electricity spot market. The main works are as following: An operation strategy of consumer electric heating equipment based on electric spot market is proposed. Besides, an operation mode which responds to the market is obtained by analysing the time span of clearing nodes in power spot market and start-stop characteristics of distributed heat load equipment. Based on this, the operating cost control function of consumer electric heating load is designed, making it possible that the load follows changes of the clearing price under the premise of ensuring that the user's heat demand is met within a day. Then we had a discussion about the impact of parameter changes on the operating strategy. With the help of the constraints of existing power grid, the operation strategy model of consumer electric heating equipment based on power spot market is realized.
\end{abstract}

\section{Introduction}

"Coal to electricity" is a national demand, and the current "coal to electricity" project cannot be separated from the strong promotion of local governments. In addition to the investment in infrastructure and corresponding grid supporting facilities, the government also needs to bear operating subsidies during the heating season each year. Excessive government investment also represents the risk of counter-substitution in some "coalto-electricity" projects.

The electricity market has sufficient benefits. Compared with the current three-stage electricity price of peak-flat-valley type and single electricity price, the electricity price in the electricity market has the characteristics of high price change frequency and huge peak-valley difference. If users can participate in the market and have the ability to respond to changing electricity prices, they can make full use of the flexibility of part load to achieve diversified electricity demand.

Electric heating load is a load with transferable characteristics. Its characteristics mainly come from two aspects, one is its own enclosure structure, and the other comes from various heat storage equipment that may be installed. Actually, the portion of users participating in electricity market is not quite good. Even in the current pilot operation of electricity market, most users cannot go to the volatile electricity market and to find out what it is. In order to solve the problem of low participation of users, the author proposes a load response power market operation model based on the user's thermal load characteristics.

\section{Node model construction}

With the help of thermal inertia of the building load, load building nodes with a certain level of enclosure can work well without physical heat storage equipment. Consequently, there is a period that the room temperature changing from temperature after cutting off heating to the lower limit of set temperature, which means this type of heating load has transferable characteristics similar to the installation of physical heat storage equipment. Besides, as they are unrelated and independent from each other, they can be linearly added.

The model of a single user node is shown in Figure 1:

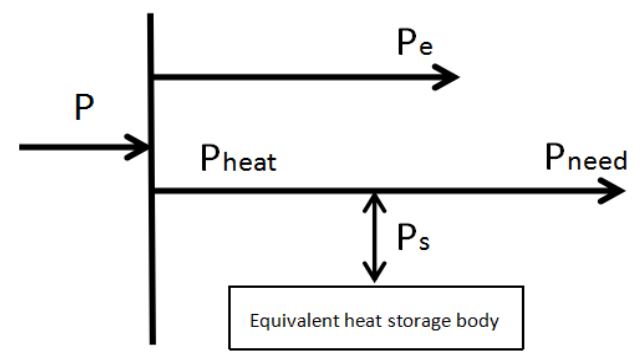

Fig. 1. Schematic diagram of user node model

\footnotetext{
* Corresponding author: 15269224539@163.com
} 
As we can see in table 1-1, the node is divided into two parts. The first one is the point connected to the power grid, which can be used to measure the power consumption plan of users. Then, the variable $\mathrm{P}$ will be used in the actual power flow calculation, which represents the actual power consumption of users. Actually, electricity load of general users is divided into two categories. One is the electrical load converted from user's heat demand corresponding to the use of electric heating equipment (so called thermal load) represented by $P_{\text {heat }}$, and the other is the user's electricity load corresponding to the remaining electrical equipment except heating equipment represented by $P_{e} . P_{\text {need }}$ is a kind of electric power corresponding to the thermal power required by users in a certain period of time, which refers to the electric power stabilizing temperature at a lower boundary of the node load temperature when the thermoelectric load starts with this electric power. It can be given by load forecasting[1].

The electric heating equipment currently used in the market is divided into two major categories from its operating characteristics, namely fixed frequency equipment and variable frequency equipment. Fixed frequency equipment is not recommended to be used in new projects due to its excessive loss, while the current equipment is mostly frequency conversion, meaning that the load of node no longer controls room temperature with start-stop rules of the fixed-frequency equipment characteristics, but can carry out continuous power adjustment within a certain range under the conditions allowed by equipment. It is not only conducive to the optimization of power consumption, but also can reduce the risk of load on security of power grid. In this model, we use $P_{s}$ to represent the variable, showing the influence on node equivalent heat storage body energy $E s$ carried by equipment operating power.

\section{Node equivalent heat storage capacity calculation}

If a load node has physical heat storage and is supported by a certain level of maintenance structure, the above research can conclude that the temperature change of room is only directly related to its terminal heat dissipation equipment, while the capacity of heat storage body installed does not directly affect it. With the help of such thesis that the load characteristics of node user load upon thermal inertia and heat storage capacity, we can equate an energy parameter $E s$, which represents the transferability characteristics of the node. This means that no matter what kind of user node it is, it is directly replaced by an equivalent variable without considering its installed equipment and other factors.

The calculation of Es energy parameter of node equivalent heat storage body is as following: The parameter is used as a measure of heat storage capacity of load node, which contains two independent parts, namely the maintenance structure parameter of node and heat storage body parameter. As is shown in Figure 2, we can use schematic diagram of fixed frequency equipment operation and room temperature to illustrate the changes without installing physical heat storage equipment[2].

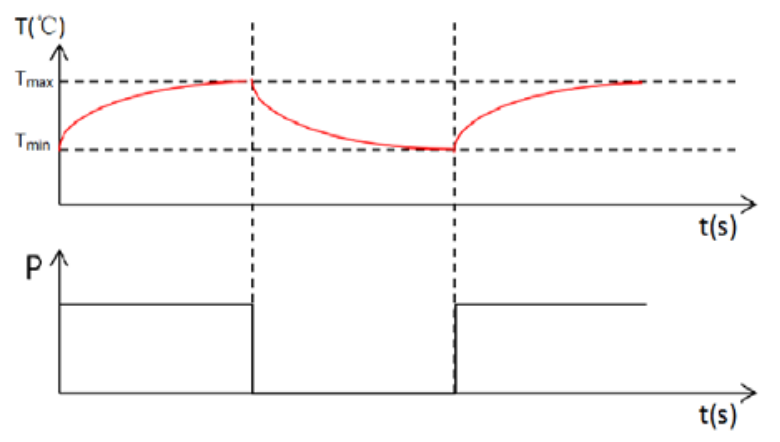

Fig.2.Schematic diagram on temperature changes of user node when fixed frequency device is turned on

We can find that the curve of room temperature drop is almost irrelevant to the heating equipment during the period of cutting off this equipment. The only factors affecting the temperature of node at this time are enclosure structure of house itself and external interference. The characteristic of this curve can just represent the thermal inertia part of the node equivalent heat storage body as well.

The model of electric heating equipment load curve combined with the maintenance structure has been arising in engineering, and its thermodynamic change process has been simplified while retaining its thermodynamic characteristics[3,4]. The simplified model is shown in Figure 3.

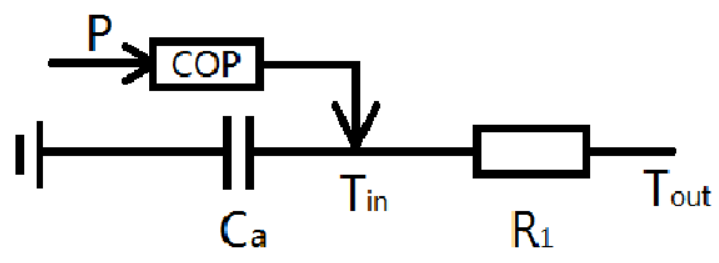

Fig.3. Schematic diagram of the first-order

In this figure, electric power $\mathrm{P}$ is converted into thermal energy by a thermoelectric conversion coefficient A in the user's electric heating device[1]. The specific heat capacity of indoor air is represented by $\mathrm{Ca}$. The indoor temperature Tin and outdoor temperature Tout establish the mathematical relationship of radiant heat dissipation through the thermal resistance $\mathrm{R} 1$. According to the law of conservation of energy, the heat entering user node in a unit time is conformed with following equation:

$$
C_{a} \frac{d T_{\text {in }}}{d t}=P A-\frac{T_{\text {in }}-T_{\text {out }}}{R_{1}}
$$

The thermal storage device model is consistent with the above model where there is a thermal storage device in user node, as the "outdoor temperature" of thermal storage device is equivalent to indoor temperature of user load. Consequently, the differential equation form of the user thermoelectric coupling model can be expressed as: 


$$
\begin{gathered}
T_{\text {in }}^{t+1}=T_{\text {out }}^{t+1}-\left(T_{\text {out }}^{t+1}-T_{\text {in }}^{t}\right) \times e^{-\frac{\Delta t}{R_{1} C_{a}}} \\
T_{\text {in }}^{t+1}=T_{\text {out }}^{t+1}+P A R_{1}-\left(T_{\text {out }}^{t+1}+P A R_{1}-T_{\text {in }}^{t}\right) \times e^{-\frac{\Delta t}{R_{1} C_{a}}}
\end{gathered}
$$

Equation 2 and Equation 3 are the mathematical relationship between temperature and time when the equipment is shut down and started.

The temperature drop curve can be obtained by fitting data of specific nodes. We can assume that at a certain moment the temperature of node (without installation of physical heat storage equipment) is at upper limit of the set temperature, and device is turned off at this time. The period that the node drops from upper temperature limit to lower boundary can be calculated by the above engineering application formula, which can represent the maximum transferability of user node, so-called the maximum value of node equivalent heat capacity. As we have defined $\mathrm{P}_{\text {need }}$ before, now it can be considered that the heating effect achieved by a node when the temperature naturally drops from the upper limit to the lower temperature limit is the same as the heating effect achieved by node electric heating equipment using $\mathrm{P}_{\text {need }}$ for heating at the same node under the same external conditions. That is, the following equation can be established. $\Delta \mathrm{t}$ is the time scale of interval between two simulation steps. $n$ is the number of simulation time steps elapsed from upper limit to lower limit of temperature.

$$
\left\{\begin{array}{c}
T_{\text {in }}^{t+1}=T_{\text {out }}^{t+1}-\left(T_{\text {out }}^{t+1}-T_{\text {in }}^{t}\right) \times e^{-\frac{\Delta t}{R_{1} C_{a}}} \\
P_{\text {need }} \times n \times \Delta T=E s m a x
\end{array}\right.
$$

The maximum equivalent heat storage capacity of node in the absence of physical heat storage equipment can be calculated after equivalent processing. If there is a physical heat storage device, the upper limit of its node equivalent heat storage capacity is sum of the maximum equivalent heat storage capacity of no physical heat storage device and the upper limit of physical heat storage body itself Qsmax under the same conditions at the node, that is:

$$
\left\{\begin{array}{l}
T_{\text {in }}^{t+1}=T_{\text {out }}^{t+1}-\left(T_{\text {out }}^{t+1}-T_{\text {in }}^{t}\right) \times e^{-\frac{\Delta t}{R_{1} C_{a}}} \\
P_{\text {need }} \times n \times \Delta T+Q \text { smax }=E s m a x
\end{array}\right.
$$

\section{Node operation optimization model of spot market}

\subsection{Objective function}

In node operation optimization model based on the spot market, the load of users is divided into two categories, which has been mentioned in the input parameters of electricity market. Actually, it is thermal load with transferability characteristics that would arouse users to be in response to the price of the spot market. While, the electrical load other than thermal load is considered as a rigid load in this operational optimization model, without any consideration of existing energy storage equipment, act as an input parameter. The operating cost objective function of users after Electricity Spot Market is as following. The total operating cost of all users in the region should be minimized with surely fulfilling their needs within a market cycle, at the same time, various operating indicators of power grid should be met.

We can establish following objective function:

$$
\min \sum_{i=1}^{T} \sum_{i=1}^{N} C\left(t_{i}\right)_{j} \times\left(P_{\text {heat }}\left(t_{i}\right)_{j}+P_{e}\left(t_{i}\right)_{j}\right) \times \Delta T \text { (6) }
$$

Among them, the parameter $\mathrm{T}$ is the total number of clearings in a market cycle. The parameter $\mathrm{N}$ is the number of load nodes in the optimization area. $C\left(t_{i}\right)_{j}$ is the real-time electricity price determined by the dayahead market clearing during the period from time $t_{i}$ to time $t_{i+1}$. $P_{\text {heat }}\left(t_{i}\right)_{j}$ is the actual heat load power of the corresponding node at corresponding time. $P_{e}\left(t_{i}\right)_{j}$ is the actual Electric load power of the corresponding node at corresponding time. $\Delta T$ is the period between these two clearing prices. Since the author extracts thermal load from user load as a variable, the electrical load left will not follow changes of the thermal load. However, it is supposed to be used in objective function in order to reflect the consistency of load model.

\subsection{Optimization principle}

Although rigid electrical load cannot be adjusted, the electrical load plays a role in constructing the basic power flow in the optimization model. It is basis for optimization calculation to construct basic power flow. As node voltage needs to maintain a stable reactive power flow during the process of power supply, the author uses DC power flow algorithm to reflect these characteristics in order to reflect the speed and simplicity of optimization calculation as well.

Thermal load of each node can be adjusted after construction of basic power flow. The principle of thermal load regulation is that each time electricity price is cleared, the signal of electricity price change is used as the driving force to adjust heat load according to the node electricity price drive. As for a specific node in a period of time, the principal diagram is as follows.

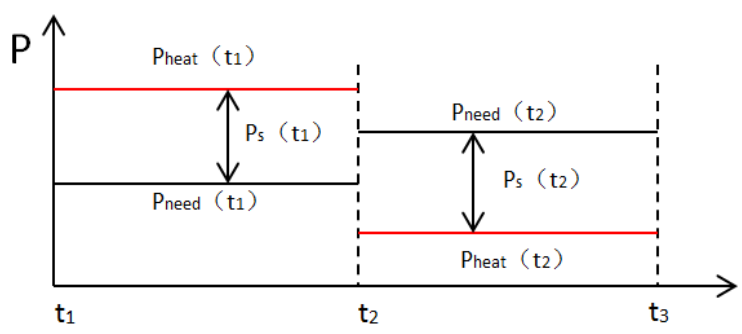

Fig.4. Single user node based on the optimization principle of day-ahead market

As is shown in Figure 4, there are three times $\mathrm{t} 1, \mathrm{t} 2$, and $\mathrm{t} 3$, and each time corresponds to a market clearing node, calculated from the market model at corresponding time. The electricity price does not change from one clearing node to the next, which is consistent with reality. The actual electricity market will be cleared every 15 
minutes within an hour. Although the four clearing prices are different, the market tends to average the four clearing prices at one time in order to reduce amount of settlement.

According to the law of market clearing, the power consumption of equipment is changed in response to electricity price at each clearing time, that is, the parameter $P_{\text {need }}\left(t_{i}\right)_{j}$ becomes a variable $P_{\text {heat }}\left(t_{i}\right)_{j}$. It can be found that there is a difference between the modulated $P_{\text {heat }}\left(t_{i}\right)_{j}$ of thermal load at this time and the $P_{\text {need }}\left(t_{i}\right)_{j}$ obtained when load forecast is carried out. This difference is determined by the operation mode of equipment. When the $P_{\text {heat }}\left(t_{i}\right)_{j}$ after strategy optimization is greater than $P_{\text {need }}\left(t_{i}\right)_{j}$, it means that the new operation mode is charging equivalent heat storage body of the node under the premise of meeting the basic heating demand of user. Similarly, if the $P_{\text {heat }}\left(t_{i}\right)_{j}$ after strategy optimization is less than $P_{\text {need }}\left(t_{i}\right)_{j}$, it can be considered that this operation mode does not meet user's basic heating demand, and it needs to be supplemented by the energy stored in node equivalent heat storage body, which is equivalent to consume the equivalent energy stored by energy storage in user node. From the above analysis, it can be known that the amount of change in the node's equivalent energy storage value during operation optimization is related to the operation strategy $P_{\text {heat }}\left(t_{i}\right)_{j}$ of the clearing node corresponding to the current time period and the predicted heat load $P_{\text {need }}\left(t_{i}\right)_{j}$.

\subsection{Constraint analysis}

The total heat load corresponding to $P_{\text {heat }}$ of node in a day-ahead market cycle should be equal to the total heat load corresponding to strategy $P_{\text {heat }}$ provided after operation optimization. That is:

$$
\sum_{t}^{T} \sum_{i}^{n} P_{\text {need }}\left(t_{i}\right)_{j} \times \Delta T=\sum_{t}^{T} \sum_{i}^{n} P_{\text {heat }}\left(t_{i}\right)_{j} \times \Delta T
$$

Because the period of users' electricity consumption (one-day each) corresponds to the opening period of the previous market, the amount of heat needed a day is a fixed value, which is determined by the previous day's heat load curve. After optimization, the total heat supply must be equal to it. Of course, this is related to the optimization cycle we choose. If several market opening time periods are selected for optimization, uniquely, this equation may not be satisfied with opening time, but it does generally.

Next, the author will explain the relationship between $P_{\text {heat }}, P_{\text {need }}$ and $P_{s}$.In this model, $P_{\text {heat }}$ is an adjustable variable connected to power grid, while $P_{\text {need }}$ is an input parameter based on heat load forecasting, and $P_{s}$ is a variable related to the heating equipment installed at user node. The relationship between them is as following:

$$
P_{\text {heat }}\left(t_{i}\right)_{j}-P_{\text {need }}\left(t_{i}\right)_{j}=P_{s}\left(t_{i}\right)_{j}
$$

The significance of this equation is that if all the equivalent heat storage capacity of room is not considered, the power supply capacity needs to be matched with the heat load demand all the time, that is, the working condition of heating equipment must be based on user's demand. When the user node is in this situation, it means that the thermal load at this time is a rigid and uninterrupted transfer load. If the concept of user equivalent heat storage body mentioned above is introduced, which is similar to the concept of energy storage equipment, the user's thermal load demand can be supplemented from two aspects. One is to supply heat with original equipment, and the other part is to be supplemented by the heat stored in equivalent heat storage body. At this time, an unequal relationship can be drawn, because in the definition of equivalent heat storage, the energy change in equivalent energy storage device actually depends on the heating equipment installed by users, so the inequality is supposed as follows:

$$
-P_{\text {need }}\left(t_{i}\right)_{j} \leq P_{s}\left(t_{i}\right)_{j} \leq P_{\text {maxj }}-P_{\text {need }}\left(t_{i}\right)_{j}
$$

Among them, $P_{\operatorname{maxj}}$ is related to the maximum power of starting heating equipment..

The existing energy of node equivalent heat storage equipment also has a limit. When the clearing node operation strategy is determined, the effect on equivalent heat storage energy value of node will continue until the next clearing time, and at the same time, a cumulative energy value from clearing time to current clearing time will be accumulated. Expressed as:

$$
\begin{gathered}
P_{s}\left(t_{i}\right)_{j} \times \Delta T=\Delta E s\left(t_{i}\right)_{j} \\
-E s_{\max j} \leq \Delta E s\left(t_{i}\right)_{j} \leq E s_{\max j} \\
0 \leq \operatorname{Es}\left(t_{i-1}\right)_{j}+\Delta E s\left(t_{i}\right)_{j} \leq E s_{\max j}
\end{gathered}
$$

Among them, $-E s_{\max j}$ is the maximum value of node equivalent heat storage calculated according to the method in Section 3.

The above constraints are all for a single node. For the single node, a set of optimization schemes can be derived mathematically, but if there are several nodes in the same area, they are coupled with each other through grid topology. If the operation is still carried out according to the optimization strategy of a single node at this time, it is likely to cause a centralized concurrency problem due to the existence of branch constraints. Therefore, during the process of optimizing strategy, it is necessary to consider the problems raised by coupling nodes on branches. In the previous algorithm construction section, the author mentioned a transfer coefficient method that is widely used in power dispatch, which uses the method originally aimed at generating nodes at load nodes[5,6]. First of all, node load is divided into two categories. The basic power flow is constructed by the equipment except heat load, and the heat load part plays a role of an adjustable variable in this model. When the heat load is superimposed on the node of basic load flow, the power flow of entire network will be redistributed. And the change of node can be reflected to each branch through transfer coefficient matrix, and then superimposed with the existing basic load flow, while new power flow distribution is obtained. It can be expressed as: 


$$
G_{k-j} \times P_{\text {heat }}\left(t_{i}\right)_{j}=\Delta P_{k}\left(t_{i}\right)
$$

In the formula, $G_{k-j}$ is the power transfer matrix of the $\mathrm{j}$ node to $\mathrm{k}$ branch, and $\Delta P_{k}$ is the power flow change of branch $\mathrm{k}$ corresponding to the change of the thermal load of node $\mathrm{j}$ from $P_{e}\left(t_{i}\right)_{j}$ to $P_{\text {heat }}\left(t_{i}\right)_{j}+P_{e}\left(t_{i}\right)_{j}$, this amount of change needs to be superimposed with the original trend. Then, the newly formed power flow is constrained, meaning the branch power flow change caused by node change cannot exceed its limit. The limitation of this branch is mainly reflected by line transformer, or some rural areas, where the current carrying capacity is limited by the low integrity of power grid construction.

$$
-P_{L \operatorname{maxk}} \leq \Delta P_{k}\left(t_{i}\right)+P_{k}\left(t_{i}\right) \leq P_{L \operatorname{maxk}}
$$

$P_{L \text { maxk }}$ is the maximum power constraint corresponding to the $\mathrm{k}$ branch, which can be calculated from the current carrying capacity.

\section{Case analysis of node operation optimization in spot market}

The main wiring of system is shown in Figure 5. The parameter settings of line parameters and bus load at a certain moment are shown in the table below. Among them, reference value of three-phase power is $\mathrm{S}_{\mathrm{B}}=100 \mathrm{MW} \cdot \mathrm{A}$, and the reference value of line voltage is $\mathrm{V}_{\mathrm{B}}=10 \mathrm{kV}$. Bus load given in the table is total power load of node without heating equipment. The heat load data has been calculated according to the method in Literature [1].

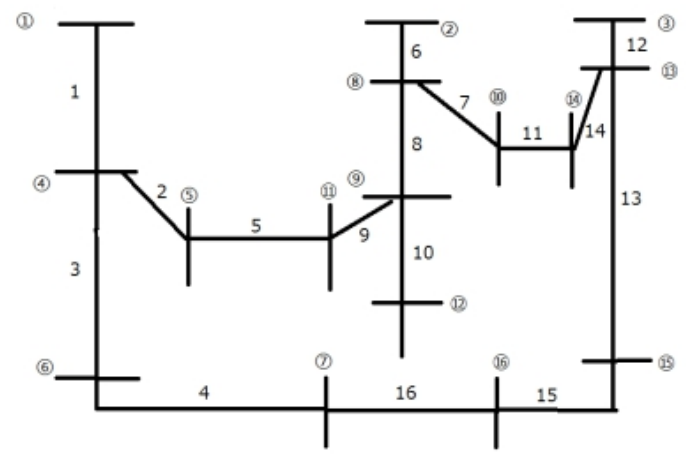

Fig.5.Network topology diagram of three-feeder system

Table 1.Line parameters of three-feeder system and related node loads

\begin{tabular}{|c|c|c|c|c|}
\hline $\begin{array}{c}\text { Branch } \\
\text { number }\end{array}$ & $\begin{array}{c}\text { First } \\
\text { node }\end{array}$ & $\begin{array}{c}\text { End } \\
\text { node }\end{array}$ & Reactance & $\begin{array}{c}\text { Active } \\
\text { power end }\end{array}$ \\
\hline 1 & 1 & 4 & 0.10 & 2.00 \\
\hline 2 & 4 & 5 & 0.11 & 3.00 \\
\hline 3 & 4 & 6 & 0.18 & 2.00 \\
\hline 4 & 6 & 7 & 0.04 & 1.00 \\
\hline 5 & 5 & 11 & 0.04 & \\
\hline 6 & 2 & 8 & 0.11 & 4.00 \\
\hline 7 & 8 & 10 & 0.11 & 1.00 \\
\hline 8 & 8 & 9 & 0.11 & 5.00 \\
\hline 9 & 9 & 11 & 0.11 & 0.60 \\
\hline 10 & 9 & 12 & 0.11 & 4.50 \\
\hline
\end{tabular}

\begin{tabular}{|c|c|c|c|c|}
\hline 11 & 10 & 14 & 0.04 & \\
\hline 12 & 3 & 13 & 0.11 & 1.00 \\
\hline 13 & 13 & 15 & 0.11 & 1.00 \\
\hline 14 & 13 & 14 & 0.12 & 1.00 \\
\hline 15 & 15 & 16 & 0.04 & 2.10 \\
\hline 16 & 7 & 16 & 0.12 & \\
\hline
\end{tabular}

Carry out the operation strategy optimization operation to obtain optimization result. lines and symbols on the figure. Carry out the operation strategy optimization operation to obtain optimization result.

\section{Optimize the heat load}

demand of some nodes before optimization

0.5

0

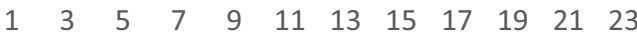

$\longrightarrow$ node $4 \longrightarrow$ node $5 \longrightarrow$ node 6

node 7 node 8

node 9

Fig.6. Heat load demand of some nodes before 16-node optimization

\section{Operation strategy of some nodes after optimization}

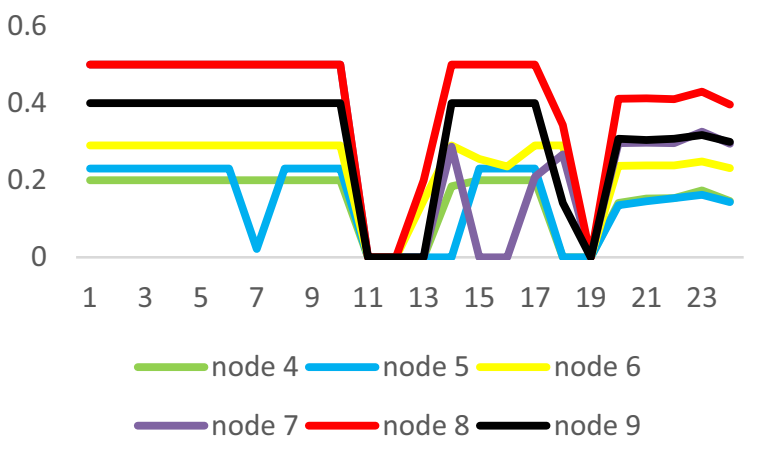

Fig.7. 16-node users, some nodes user's optimized operating strategy

The equivalent heat storage results of some load node are as follows: 


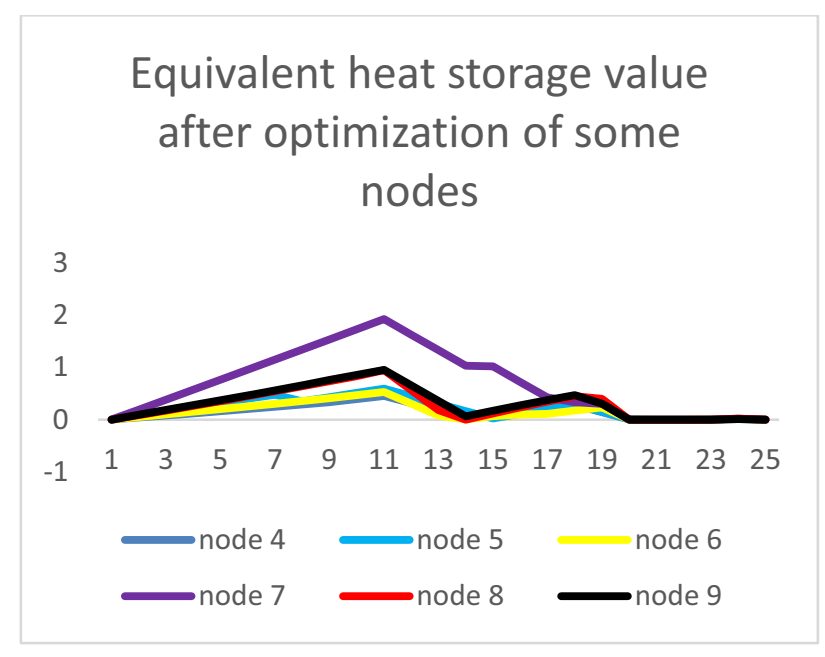

Fig.7. Equivalent heat storage value after optimization of some nodes

\section{Summary}

This paper proposes a heating equipment operation optimization model based on the power spot market, and optimizes the power consumption strategy of the nodes that use electric heating users. Load aggregators or electricity sales companies can make full use of the transferable characteristics of heat loads while ensuring the safety of the regional power grid, reducing the operating costs of electric heating equipment throughout the region. With the gradual implementation of crossregional power trading, electric heating loads can also carry out diversified market transactions based on their own load characteristics.

\section{References}

1. Qin Yu,Jiang Limin, Yu Xiaoling,Guo Bingqing. Study and application on accurate forecasting model of power load based on thermal conditions of power-heat equipment [J].Power Demand Side Management,2020,22(03):8-13.

2. Fan Shuai, Jia Kunqi, Guo Bingqing, Jiang Limin, Wang Zhihua, He Guangyu. Collaborative Optimal Operation Strategy for Decentralized Electric Heating Loads [J]. Automation of Electric Power Systems, 2017,41(19):20-29.

3. N. Lu, "An Evaluation of the HVAC Load Potential for Providing Load Balancing Service," in IEEE Transactions on Smart Grid, vol. 3, no. 3, pp. 12631270, Sept. 2012.

4. S. Katipamula and N. Lu, "Evaluation of residential HVAC control strategies for demand response programs," ASHRAE Trans., vol. 1, no. 12, pp. 112, 2006.

5. Huang Zerong, Peng Jianchun, Xiao Wenxian, Dong Kai, Gong Yanping. On the rationality and optimality of GSDF and GGDF [J]. Power System Protection and Control,2009,37(21):7-10+40
6. Shen Yu, Xia Qing, Kang Chongqing. generation jointly shift distribution factor (gjsdf) and faster security analysis algorithm in economic dispatch [J]. Automation of Electric Power Systems,2003(18):1317. 